\title{
Multiband Differential Modulation for UWB Communication Systems ${ }^{\dagger}$
}

\author{
Thanongsak Himsoon, Weifeng $\mathrm{Su}^{\ddagger}$, and K. J. Ray Liu
}

Department of Electrical and Computer Engineering, University of Maryland, College Park, MD 20742.

${ }^{\ddagger}$ Department of Electrical Engineering, State University of New York (SUNY) at Buffalo, Buffalo, NY 14260.

\begin{abstract}
In this paper, we propose a differential encoding and decoding scheme for multiband UWB systems. The proposed scheme incorporates frequency-domain differential en/decoding with the hopping multiband OFDM modulation. To capture the effect of multipath-rich clustering property of UWB channels, we characterize the pairwise error probability performance of the proposed scheme in terms of cluster and ray arrival rates. It turns out that the diversity advantage does not strongly depend on the random-clustering of UWB channels, and we can achieve the same diversity gain in different channel environments. However, the system performance relies on the clustering behavior through the coding gain. Simulation results show that the proposed differential scheme achieves good performance in the shortrange line-of-sight scenarios. In addition, the jointly encoded differential multiband UWB scheme is able to yield superior performance to the uncoded coherent multiband UWB system at high SNR.
\end{abstract}

\section{INTRODUCTION}

Ultra-wideband (UWB) is an emerging technology that offers great promises to satisfy the growing demand for low cost and high-speed digital wireless home networks. A traditional UWB technology, which occupies the available bandwidth of $7.5 \mathrm{GHz}$, is based on single-band approaches that directly modulate data into a sequence of impulse-like waveforms. Recently, multiband UWB schemes were proposed [1], in which the entire UWB frequency band is divided into several subbands, each with a bandwidth of at least $500 \mathrm{MHz}$. Since many applications enabled by UWB are expected to be in portable devices, low complexity becomes a fundamental requirement. This indicates the important need of a simple transceiver design. In conventional coherent detection system, it requires channel estimation and hence introduces complexity to the receiver. An alternative approach to overcome such problem is through the use of non-coherent detection techniques. In recent years, non-coherent UWB systems have been proposed, e. g. in [2]. Nevertheless, most of the existing works, are based on single-band impulse radio technology. The current works for multiband UWB mostly focus on coherent detection schemes [3].

Differential space-time modulation [4] has been widely accepted as one of many practical alternatives that bypasses multi-channel estimation and provides a good tradeoff between performance and complexity in frequency-non-selective multiple-input multiple-output (MIMO) systems. In order to further improve the performance and gain full space-frequency diversity in wideband systems, differential modulation incorporating with MIMO-OFDM transmission has been introduced in [5]-[7] and references therein. The scheme in [5]-[6] focuses on time-domain differential encoding, while that in [7] and

\footnotetext{
${ }^{\dagger}$ This work was supported in part by U.S. Army Research Laboratory under Cooperative Agreement DAAD 190120011.
}

some part of [5] are based on frequency-domain differential encoding.

In this paper, we propose a differential encoding and decoding scheme for UWB systems employing MIMO multiband OFDM. In the proposed scheme, the information is jointly encoded across spatial, temporal, and frequency domains. By differentially en/decoding in the frequency domain, the proposed scheme does not rely on the assumption that the fading channel stays constant within several OFDM symbol durations. In this way, we are able to explore the available space and frequency diversities, richly inherent in UWB channels. More importantly, it allows us to incorporate the differential transmission with hopping multiband OFDM modulation so as to gain the additional diversity from time-domain spreading. In order to capture the unique multipath-rich and randomclustering properties of UWB channels, we characterize the pairwise error probability performance of the proposed scheme based on the Saleh-Valenzuela (S-V) fading model. Finally, the merit of our proposed scheme is shown through computer simulations.

\section{SySTEM MODEL}

We consider a peer-to-peer multiband UWB system equipped with $M_{t}$ transmit and $M_{r}$ receive antennas. Within each subband, OFDM modulation with $N$ subcarriers is used at each transmit antenna. The modulated OFDM symbols can be time-interleaved across several subbands as specified in [1].

According to the IEEE 802.15.3a standard [8], the fading channels for UWB systems are based on the S-V model for indoor channels [9]. The mathematical model of the channel impulse response from the $i^{\text {th }}$ transmit antenna to the $j^{\text {th }}$ receive antenna during the $k^{t h}$ OFDM block is given by [8]

$$
h_{i j}^{k}(t)=\sum_{c=0}^{C} \sum_{l=0}^{L} \alpha_{i j}^{k}(c, l) \delta\left(t-T_{c}-\tau_{c, l}\right),
$$

where $i=1, \cdots, M_{t}$ and $j=1, \cdots, M_{r}$. In each $i-j$ transmission link, $\alpha_{i j}^{k}(c, l)$ denotes the multipath gain coefficient of the $l^{t h}$ arrival in the $c^{t h}$ cluster at time $k$. The time duration $T_{c}$ represents the arrival time of the $c^{t h}$ cluster, and $\tau_{c, l}$ is the delay of the $l^{\text {th }}$ path in the $c^{\text {th }}$ cluster relative to the cluster arrival time $T_{c}$. The cluster arrivals and the path arrivals within each cluster are modelled by Poisson process with rate $\Lambda$ and $\lambda(\lambda>\Lambda)$, respectively. The path amplitude $\left|\alpha_{i j}^{k}(c, l)\right|$ may follow the log-normal, Nakagami or Rayleigh distributions [8], whereas the phase $\angle \alpha_{i j}^{k}(c, l)$ is uniform random variable over $[0,2 \pi)$. In this paper, $\left|\alpha_{i j}^{k}(c, l)\right|$ is modeled as Rayleigh distribution, i.e., $\alpha_{i j}^{k}(c, l)$ are zero-mean complex Gaussian random variables with variances [8]

$$
\Omega_{c, l}=\mathrm{E}\left[|\alpha(c, l)|^{2}\right]=\Omega_{0,0} \exp \left(-\frac{T_{c}}{\Gamma}-\frac{\tau_{c, l}}{\gamma}\right),
$$


where $\Omega_{0,0}$ is the mean energy of the first path of the first cluster, $\Gamma$ is the cluster decay factor, and $\gamma$ is the ray decay factor. The channel coefficients are assumed to be spatially uncorrelated and the powers of all independent delay paths are normalized such that $\sum_{c=0}^{C} \sum_{l=0}^{L} \Omega_{c, l}=1$. The channel model parameters corresponding to different scenarios are provided in [1].

We denote $x_{i}^{k}(n)$ as a differentially encoded data symbol to be transmitted on the $n^{\text {th }}$ subcarrier at the $i^{\text {th }}$ transmit antenna during the $k^{\text {th }}$ OFDM symbol period. At the receiver, after cyclic prefix removing and OFDM demodulating, the received signal at the $n^{\text {th }}$ subcarrier at the $j^{\text {th }}$ receive antenna during the $k^{\text {th }}$ OFDM block is given by

$$
y_{j}^{k}(n)=\sqrt{\rho} \sum_{i=1}^{M_{t}} x_{i}^{k}(n) H_{i j}^{k}(n)+w_{j}^{k}(n),
$$

where $\rho$ is the average signal to noise ratio per receiver, and

$$
H_{i j}^{k}(n)=\sum_{c=0}^{C} \sum_{l=0}^{L} \alpha_{i j}^{k}(c, l) \exp \left[-\mathbf{j} 2 \pi n \Delta f\left(T_{c}+\tau_{c, l}\right)\right]
$$

is the subchannel gain. Here, $\Delta f=1 / T_{s}$ is the inter-subcarrier spacing, and $T_{s}$ is the OFDM symbol period. The additive noise $w_{j}^{k}(n)$ is modeled as independent complex Gaussian random variable with zero mean and unit variance.

\section{The PRoposed DifFERENTIAL SCHEME FOR MULTIBAND UWB SYSTEMS}

We propose in this section a frequency-domain differential scheme for multiband UWB system. In addition, we exploit the additional diversity from band hopping inherently in multiband transmission by jointly encoding across $K$ OFDM blocks and transmitted the $K$ OFDM symbols on different subbands. In each OFDM block, we exploit subcarrier interleaving strategy as in [7].

\section{A. Transmit Signal and Differential Encoding Structures}

We introduce a differential multiband UWB scheme based on a transmit signal structure proposed in [3]. Particularly, $\mathbf{X}$ is a jointly design $K N \times M_{t}$ space-time-frequency code structure in which it consists of stacking space-frequency signal $\mathbf{X}^{k}$, each of dimension $N \times M_{t}$, for $K$ OFDM symbols. To reduce complexity of the design, we divide $\mathbf{X}^{k}$ into serval submatrices or groups. By introducing a fixed integer $G(1 \leq G \leq N)$ as a number of jointly encoded subcarriers, $\mathbf{X}^{k}$ at each OFDM symbol is partitioned into $P=\left\lfloor N /\left(G M_{t}\right)\right\rfloor$ submatrices as follows [3]:

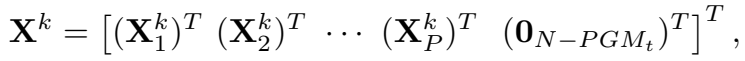

for $k=1,2, \cdots, K$ and $T$ denotes the matrix transposition. The $\left(N-P G M_{t}\right) \times M_{t}$ matrix $\mathbf{0}_{N-P G M_{t}}$ represents a zero padding matrix to be inserted if $N$ cannot be divided by $G M_{t}$. Each of the $G M_{t} \times M_{t}$ submatrix $\mathbf{X}_{p}^{k}$, for $k=1, \cdots, K$, and $p=1,2, \ldots, P$, is modeled as

$$
\mathbf{X}_{p}^{k}=\operatorname{diag}\left(\mathbf{x}_{p, 1}^{k} \mathbf{x}_{p, 2}^{k} \cdots \mathbf{x}_{p, M_{t}}^{k}\right)
$$

where $\operatorname{diag}(\cdot)$ denotes diagonal operation that places all vectors or scalar elements at the main diagonal matrix, and $\mathbf{x}_{p, i}^{k}$, for $i=1,2, \ldots, M_{t}$, is a $G \times 1$ vector:

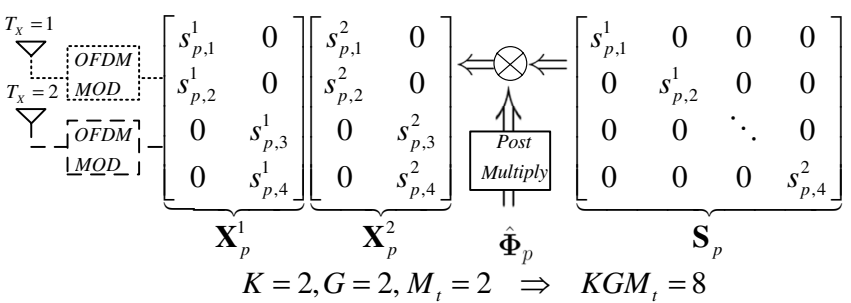

Fig. 1: Example of differential encoded signal matrix and transmit signal structure for the UWB system employing multiband OFDM, $K=2, G=2$, and $M_{t}=2$.

$$
\mathbf{x}_{p, i}^{k}=\left[\begin{array}{llll}
s_{p,(i-1) G+1}^{k} & s_{p,(i-1) G+2}^{k} & \cdots & s_{p, i G}^{k}
\end{array}\right]^{T},
$$

in which all $s_{p, m}^{k}, m=1,2, \ldots, G M_{t}$, are differentially encoded symbols that will be specified later. We will differentially encode across $K$ OFDM symbols within each group, and our desired transmit signal structure for the $p^{t h}$ group after differentially encoding is $K G M_{t} \times M_{t}$ matrix:

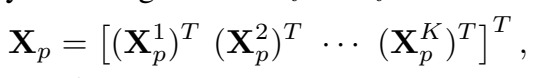

in which the $i^{\text {th }}$ column contains encoded symbols to be transmit at the $i^{\text {th }}$ transmit antenna.

We now specify information matrices to be differentially encoded as follows. Let $\mathbf{V}_{p}$ denote a $K G M_{t} \times K G M_{t}$ unitary information matrix having diagonal form as

$$
\mathbf{V}_{p}=\operatorname{diag}\left(\left[\begin{array}{llll}
v_{p, 1} & v_{p, 2} & \cdots & v_{p, K G M_{t}}
\end{array}\right]^{T}\right),
$$

in which $v_{p, m}$ is an information symbol. We will jointly design the data within each information matrix $\mathbf{V}_{p}$, but independently design the matrices $\mathbf{V}_{p}$ 's for different $p$.

Let $\mathbf{S}_{p}$ be a $K G M_{t} \times K G M_{t}$ differentially encoded signal matrix. We recursively construct $\mathbf{S}_{p}$ by [4]

$$
\mathbf{S}_{p}=\left\{\begin{array}{ll}
\mathbf{V}_{p} \mathbf{S}_{p-1}, & p \geq 1 \\
\mathbf{I}_{K G M_{t},}, & p=0
\end{array} .\right.
$$

Due to the diagonal structure of $\mathbf{V}_{p}, \mathbf{S}_{p}$ can be expressed as

$$
\mathbf{S}_{p}=\operatorname{diag}\left(\left[s_{p, 1}^{1}, \cdots, s_{p, G M_{t}}^{1}, \cdots, s_{p, 1}^{K}, \cdots, s_{p, G M_{t}}^{K}\right]^{T}\right),
$$

where $s_{p, m}^{k}$ is the differentially encoded complex symbol to be transmitted at subcarrier $(p-1) G M_{t}+m$ during the $k^{t h}$ OFDM block. In order to transform $\mathbf{S}_{p}$ into (8), we introduce a $K G M_{t} \times M_{t}$ multiplicative mapping matrix

$$
\hat{\boldsymbol{\Phi}}_{p}=\mathbf{1}_{K} \otimes \boldsymbol{\Phi}_{p},
$$

where $\mathbf{1}_{K}$ denotes a $K \times 1$ vector of all ones, $\otimes$ denotes the Kronecker product [10], $\boldsymbol{\Phi}_{p}=\left[\begin{array}{llll}\phi_{1} & \phi_{2} & \cdots & \phi_{M_{t}}\end{array}\right]$ is the $G M_{t} \times M_{t}$ mapping matrix in which $\phi_{i}=\mathbf{e}_{i} \otimes \mathbf{1}_{G}$ is a $G M_{t} \times 1$ vector, and $\mathbf{e}_{i}$ is an $M_{t} \times 1$ unit vector whose its $i^{t h}$ component is one and all others are zeroes. We post-multiply $\mathbf{S}_{p}$ by $\hat{\boldsymbol{\Phi}}_{p}$, resulting in the desired $K G M_{t} \times M_{t}$ matrix

$$
\mathbf{X}_{p}=\mathbf{S}_{p} \hat{\mathbf{\Phi}}_{p}
$$

as specified in (8). For better understanding the concept of the proposed scheme, we show in Figure 1 an example of differentially encoded signals in case of $K=2, G=2$, and $M_{t}=2$.

\section{B. Differentially Decoding}

The received signal vector corresponding to the transmitted matrix $\mathbf{X}_{p}$ is given by

$$
\mathbf{y}_{p}=\sqrt{\rho}\left(\mathbf{I}_{M_{r}} \otimes \mathcal{D}\left(\mathbf{X}_{p}\right)\right) \mathbf{h}_{p}+\mathbf{w}_{p},
$$


where $\mathcal{D}\left(\mathbf{X}_{\mathbf{p}}\right)$ denotes an operation on an $K G M_{t} \times M_{t}$ matrix $\mathbf{X}_{\mathrm{p}}$ that converts each column of $\mathbf{X}_{\mathbf{p}}$ into a diagonal matrix and results in an $K G M_{t} \times K G M_{t} M_{t}$ matrix, expressed by

$\mathcal{D}\left(\mathbf{X}_{\mathbf{p}}\right)=\mathcal{D}\left(\left[\mathbf{x}_{p, 1} \cdots \mathbf{x}_{p, M_{t}}\right]\right)=\left[\operatorname{diag}\left(\mathbf{x}_{p, 1}\right) \cdots \operatorname{diag}\left(\mathbf{x}_{p, M_{t}}\right)\right]$

The matrix $\mathbf{h}_{p}=\left[\begin{array}{lll}\left(\mathbf{h}_{p, 1}\right)^{T}\left(\mathbf{h}_{p, 2}\right)^{T} & \ldots & \left(\mathbf{h}_{p, M_{r}}\right)^{T}\end{array}\right]^{T}$ is a channel matrix constructed from $K G M_{t} M_{t} \times 1$ matrix:

$\mathbf{h}_{p, j}=\left[\left(\mathbf{h}_{p, 1 j}^{1}\right)^{T} \cdots\left(\mathbf{h}_{p, 1 j}^{K}\right)^{T} \cdots\left(\mathbf{h}_{p, M_{t} j}^{1}\right)^{T} \cdots\left(\mathbf{h}_{p, M_{t} j}^{K}\right)^{T}\right]$ where $\mathbf{h}_{p, i j}^{k}=\left[H_{i j}^{k}\left((p-1) G M_{t}\right) \cdots H_{i j}^{k}\left(p G M_{t}-1\right)\right]^{T}$ is a channel gain vector of size $G M_{t} \times 1$. The received

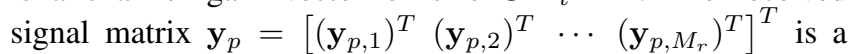
$K G M_{t} M_{r} \times 1$ matrix constructed from the $K G M_{t} \times 1$ receive signal vector $\mathbf{y}_{p, j}=\left[\left(\mathbf{y}_{p, j}^{1}\right)^{T}\left(\mathbf{y}_{p, j}^{2}\right)^{T} \cdots\left(\mathbf{y}_{p, j}^{K}\right)^{T}\right]^{T}$, in which $\mathbf{y}_{p, j}^{k}=\left[y_{j}^{k}\left((p-1) G M_{t}\right) \cdots y_{j}^{k}\left(p G M_{t}-1\right)\right]^{T}$ is a $G M_{t} \times 1$ matrix. The noise matrix $\mathbf{w}_{p}$ is in the same form as $\mathbf{y}_{p}$ with $\mathbf{y}_{p, j}$ and $\mathbf{y}_{p, j}^{k}$ replaced by $\mathbf{w}_{p, j}$ and $\mathbf{w}_{p, j}^{k}$, respectively.

By substituting (13) into (14), we can reformulate $\mathbf{y}_{p}$ as

$$
\mathbf{y}_{p}=\sqrt{\rho}\left(\mathbf{I}_{M_{r}} \otimes \mathcal{D}\left(\mathbf{S}_{p} \hat{\boldsymbol{\Phi}}_{p}\right)\right) \mathbf{h}_{p}+\mathbf{w}_{p} .
$$

To simplify (17), we first observe from (12) that $\hat{\boldsymbol{\Phi}}_{p}$ can be re-expressed as $\hat{\boldsymbol{\Phi}}_{p}=\left[\tilde{\boldsymbol{\phi}}_{1} \tilde{\boldsymbol{\phi}}_{2} \cdots \tilde{\boldsymbol{\phi}}_{M_{t}}\right]$, where $\tilde{\boldsymbol{\phi}}_{i}=\mathbf{1}_{K} \otimes \boldsymbol{\phi}_{i}$. Therefore, $\mathcal{D}\left(\mathbf{S}_{p} \hat{\boldsymbol{\Phi}}_{p}\right)$ can be given by

$$
\mathcal{D}\left(\mathbf{S}_{p} \hat{\boldsymbol{\Phi}}_{p}\right)=\left[\operatorname{diag}\left(\mathbf{S}_{p} \tilde{\boldsymbol{\phi}}_{1}\right) \cdots \operatorname{diag}\left(\mathbf{S}_{p} \tilde{\boldsymbol{\phi}}_{M_{t}}\right)\right] .
$$

According to (16) and (18) for each $j$, we have $\mathcal{D}\left(\mathbf{S}_{p} \hat{\mathbf{\Phi}}_{p}\right) \mathbf{h}_{p, j}=\sum_{i=1}^{M_{t}} \operatorname{diag}\left(\mathbf{S}_{p} \tilde{\boldsymbol{\phi}}_{i}\right) \mathbf{h}_{p, i j}$ which can be simplified to

$$
\mathcal{D}\left(\mathbf{S}_{p} \hat{\boldsymbol{\Phi}}_{p}\right) \mathbf{h}_{p, j}=\mathbf{S}_{p} \sum_{i=1}^{M_{t}} \tilde{\boldsymbol{\phi}}_{i} \circ \mathbf{h}_{p, i j} \triangleq \mathbf{S}_{p} \tilde{\mathbf{h}}_{p, j},
$$

where the last term on the right hand side results from using the property of Hadamard product [10]. The $K G \times 1$ channel matrix $\tilde{\mathbf{h}}_{p, j}$ can be obtained by substituting (16) into (19) as $\tilde{\mathbf{h}}_{p, j}=\left[\left(\tilde{\mathbf{h}}_{p, 1 j}^{1}\right)^{T} \cdots\left(\tilde{\mathbf{h}}_{p, 1 j}^{K}\right)^{T} \cdots\left(\tilde{\mathbf{h}}_{p, M_{t} j}^{1}\right)^{T} \cdots\left(\tilde{\mathbf{h}}_{p, M_{t} j}^{K}\right)^{T}\right]^{T}$, where

$$
\tilde{\mathbf{h}}_{p, i j}^{k}=\left[H_{i j}^{k}\left(n_{p, i}^{0}\right) H_{i j}^{k}\left(n_{p, i}^{1}\right) \cdots H_{i j}^{k}\left(n_{p, i}^{G-1}\right)\right]^{T}
$$

is of size $G \times 1$, and $n_{p, i}^{g}=(i-1) G+(p-1) G M_{t}+g$ for $g=0,1, \ldots, G-1$. By denoting a $K G M_{t} M_{r} \times 1$ channel gain vector:

$$
\tilde{\mathbf{h}}_{p}=\left[\left(\tilde{\mathbf{h}}_{p, 1}\right)^{T}\left(\tilde{\mathbf{h}}_{p, 2}\right)^{T} \cdots\left(\tilde{\mathbf{h}}_{p, M_{r}}\right)^{T}\right]^{T},
$$

and using (19) for all $j$, we obtain an equivalent expression

$$
\left(\mathbf{I}_{M_{r}} \otimes \mathcal{D}\left(\mathbf{X}_{p}\right)\right) \mathbf{h}_{p}=\left(\mathbf{I}_{M_{r}} \otimes \mathbf{S}_{p}\right) \tilde{\mathbf{h}}_{p} .
$$

Finally, from (22) we can simplify (17) to

$$
\mathbf{y}_{p}=\sqrt{\rho}\left(\mathbf{I}_{M_{r}} \otimes \mathbf{S}_{p}\right) \tilde{\mathbf{h}}_{p}+\mathbf{w}_{p} \text {. }
$$

For notation convenience, let us define $\mathcal{S}_{p} \triangleq\left(\mathbf{I}_{M_{r}} \otimes \mathbf{S}_{p}\right)$ and $\mathcal{V}_{p} \triangleq\left(\mathbf{I}_{M_{r}} \otimes \mathbf{V}_{p}\right)$ such that

$$
\mathcal{S}_{p}=\left(\mathbf{I}_{M_{r}} \otimes \mathbf{V}_{p}\right) \mathcal{S}_{p-1}=\mathcal{V}_{p} \mathcal{S}_{p-1} .
$$

Accordingly, using (23)-(24) and after some manipulations, we can write the received signal as

$$
\mathbf{y}_{p}=\mathcal{V}_{p} \mathbf{y}_{p-1}+\sqrt{2} \tilde{\mathbf{w}}_{p},
$$

where $\tilde{\mathbf{w}}_{p}=\frac{1}{\sqrt{2}} \mathbf{w}_{p}-\mathcal{V}_{p} \mathbf{w}_{p-1}$ is a noise vector whose each element is independent complex Gaussian random variable with zero mean and unit variance. Without acquiring channel state information, the detector follows the maximum likelihood (ML) decision rule [4]

$$
\hat{\mathcal{V}}_{p}=\arg \min _{\mathcal{V}_{p} \in \mathbb{V}_{p}}\left\|\mathbf{y}_{p}-\mathcal{V}_{p} \mathbf{y}_{p-1}\right\|_{F},
$$

where $\|\cdot\|_{F}$ denotes the Frobinius norm [10]. Even though the decoding complexity increases exponentially with $R K G M_{t}$ where $R$ is the transmission rate, the decoding complexity can be reduced to polynomial in $K G M_{t}$ by lattice reduction algorithm [11].

\section{PAIRWISE ERROR PROBABILITY}

In this section, we provide an approximate PEP formulation based on the results in [12][13]. We first note that the channel matrix in (21) can be reexpressed as $\tilde{\mathbf{h}}_{p}=\tilde{\mathbf{h}}_{p-1}+\Delta \tilde{\mathbf{h}}_{p}$, where $\Delta \tilde{\mathbf{h}}_{p}$ represents the channel mismatch between $\tilde{\mathbf{h}}_{p}$ and $\tilde{\mathbf{h}}_{p-1}$. For analytical tractability, this section confines the analysis to the case when $\Delta \tilde{\mathbf{h}}_{p}$ is negligible, i.e., $\tilde{\mathbf{h}}_{p-1} \approx \tilde{\mathbf{h}}_{p}$. Such performance formulation provides us a benchmark for subsequent performance comparisons. Later in Section V, we will show from the numerical results how the channel mismatch affects the system performance.

For specific values of $T_{c}$ and $\tau_{c, l}$ the PEP upper bound is given in ([12], proposition 7). The average PEP can be obtained by averaging over Poisson distributions, however, it is difficult if not possible to obtain the average PEP. In what follows, we use the approximation approach as in [13]. Suppose that $\mathcal{V}_{p}$ and $\hat{\mathcal{V}}_{p}$ are two different information matrices, the asymptotic PEP can be approximated as

$$
P_{a}\left(\mathcal{V}_{p} \rightarrow \hat{\mathcal{V}}_{p}\right) \approx\left(\begin{array}{c}
2 \nu-1 \\
\nu
\end{array}\right)\left(\prod_{m=1}^{\nu} \beta_{p, m}\right)^{-1}\left(\frac{\rho}{2}\right)^{-\nu},
$$

where $\rho$ is an average signal-to-noise ratio per symbol, $\nu$ is the rank and $\beta_{p, m}$ 's are the non-zero eigenvalues of the matrix

$$
\boldsymbol{\Psi}_{p} \triangleq \mathcal{S}_{p-1} \boldsymbol{\Sigma}_{\tilde{\mathbf{h}}_{p}} \mathcal{S}_{p-1}^{H}\left(\mathcal{V}_{p}-\hat{\mathcal{V}}_{p}\right)^{H}\left(\mathcal{V}_{p}-\hat{\mathcal{V}}_{p}\right)
$$

in which $\boldsymbol{\Sigma}_{\tilde{\mathbf{h}}_{p}}=E\left[\tilde{\mathbf{h}}_{p} \tilde{\mathbf{h}}_{p}^{H}\right]$ denotes the correlation matrix of channel vector $\tilde{\mathbf{h}}_{p}$.

To simplify the expression for matrix $\boldsymbol{\Psi}_{p}$ in (28), we evaluate the channel correlation matrix $\boldsymbol{\Sigma}_{\tilde{\mathbf{h}}_{p}}$ as follows. Due to the band hopping, the $K$ OFDM symbols in each signal matrix are sent over different subbands. With an ideal band hopping, we assume that the signal transmitted over $K$ different frequencybands undergo independent fading. Assuming also that the MIMO channel is spatially uncorrelated, we can find that $\boldsymbol{\Sigma}_{\tilde{\mathbf{h}}_{p}}=\mathbf{I}_{K M_{r}} \otimes E\left[\tilde{\mathbf{h}}_{p, j}^{k}\left(\tilde{\mathbf{h}}_{p, j}^{k}\right)^{H}\right]$, and it can be simplified to

$$
\boldsymbol{\Sigma}_{\tilde{\mathbf{h}}_{p}}=\mathbf{I}_{K M_{r}} \otimes \operatorname{diag}\left(\mathbf{R}_{p, 1}, \cdots, \mathbf{R}_{p, M_{t}}\right) \text {, }
$$

where $\mathbf{R}_{p, i} \triangleq E\left[\tilde{\mathbf{h}}_{p, i j}^{k}\left(\tilde{\mathbf{h}}_{p, i j}^{k}\right)^{H}\right]$ denotes the correlation matrix and it is the same for all $j^{\prime} s$. From (20), we can see that the diagonal elements, i. e., the $(u, u)^{t h}$ elements, of $\mathbf{R}_{p, i}$ are

$$
R_{p, i}^{u, u}=E\left[\left|H_{i j}^{k}\left(n_{p, i}^{u}\right)\right|^{2}\right]=E\left[\sum_{c=0}^{C} \sum_{l=0}^{L} \Omega_{c, l}\right]=1 .
$$

The off-diagonal components, i.e., the $(u, v)^{t h}$ for $u \neq v$ components, of $\mathbf{R}_{p, i}$ can be expressed as

$$
\begin{aligned}
R_{p, i}^{u, v} & =E\left[H_{i j}^{k}\left(n_{p, i}^{u}\right)\left(H_{i j}^{k}\left(n_{p, i}^{v}\right)\right)^{H}\right] \\
& =\sum_{c=0}^{C} \sum_{l=0}^{L} E\left[\Omega_{c, l} e^{-\mathbf{j} 2 \pi \Delta f\left(n_{p, i}^{u}-n_{p, i}^{v}\right)\left(T_{c}+\tau_{c, l}\right)}\right] .
\end{aligned}
$$


Observing that $n_{p, i}^{u}-n_{p, i}^{v}=u-v$ and using (2), we can re-express (31) as

$$
R_{p, i}^{u, v}=\Omega_{0,0} \sum_{c=0}^{C} \sum_{l=0}^{L} E\left[e^{-g\left(\frac{1}{\Gamma}, u, v\right) T_{c}-g\left(\frac{1}{\gamma}, u, v\right) \tau_{c, l}}\right] .
$$

where $g(a, u, v)=a+\mathbf{j} 2 \pi(u-v) \Delta f$. According to the Poisson distribution of the multipath delays, $T_{c}$ and $\tau_{c, l}$ can be modeled as summations of identically independent distributed (iid) exponential random variables with parameter $\Lambda$ and $\lambda$, respectively. Therefore, averaging (32) over the distribution of $T_{c}$ and $\tau_{c, l}$, we arrive at

$$
R_{p, i}^{u, v}=\Omega_{0,0} \sum_{c=0}^{C} \sum_{l=0}^{L} \frac{\Lambda+g\left(\frac{1}{\Gamma}, u, v\right)}{g\left(\frac{1}{\Gamma}, u, v\right)} \frac{\lambda+g\left(\frac{1}{\gamma}, u, v\right)}{g\left(\frac{1}{\gamma}, u, v\right)} .
$$

Since $R_{p, i}^{u, v}$ is the same for all $i$ 's and $p$ 's, we denote $\mathbf{R} \triangleq \mathbf{R}_{p, i}$, which allow us to further simplify (29) to

$$
\boldsymbol{\Sigma}_{\tilde{\mathbf{h}}_{p}}=\mathbf{I}_{K M_{t} M_{r}} \otimes \mathbf{R} \text {. }
$$

Substituting (34) into (28) and applying the property of tensor product $\left(\mathbf{A}_{1} \otimes \mathbf{B}_{1}\right)\left(\mathbf{A}_{2} \otimes \mathbf{B}_{2}\right)\left(\mathbf{A}_{3} \otimes \mathbf{B}_{3}\right)=\left(\mathbf{A}_{1} \mathbf{A}_{2} \mathbf{A}_{3} \otimes\right.$ $\left.\mathbf{B}_{1} \mathbf{B}_{2} \mathbf{B}_{3}\right)$, we obtain

$$
\boldsymbol{\Psi}_{p}=\mathbf{I}_{M_{r}} \otimes \boldsymbol{\Theta}_{p},
$$

in which

$$
\boldsymbol{\Theta}_{p}=\mathbf{S}_{p-1}\left(\mathbf{I}_{K M_{t}} \otimes \mathbf{R}\right) \mathbf{S}_{p-1}^{H} \boldsymbol{\Delta},
$$

and $\boldsymbol{\Delta}=\left(\mathbf{V}_{p}-\hat{\mathbf{V}}_{p}\right)^{H}\left(\mathbf{V}_{p}-\hat{\mathbf{V}}_{p}\right)$. Hence, by (35), the PEP in (27) can be expressed as

$$
P_{a}\left(\mathbf{V}_{p} \rightarrow \hat{\mathbf{V}}_{p}\right) \approx\left(\begin{array}{c}
2 r M_{r}-1 \\
r M_{r}
\end{array}\right)\left(\prod_{m=1}^{r} \lambda_{p, m}\right)^{-M_{r}}\left(\frac{\rho}{2}\right)^{-r M_{r}}
$$

where $r$ is the rank of $\boldsymbol{\Theta}_{p}$ and $\lambda_{p, m}$ 's are the non-zero eigenvalues of $\boldsymbol{\Theta}_{p}$.

To quantify the maximum diversity order which is the exponent of $\rho / 2$ in (37), we observe from (36) that $\mathbf{S}_{p-1}$ and $\mathbf{V}_{p}$ are of size $G K M_{t} \times G K M_{t}$, and the correlation matrix $\mathbf{R}$ is of size $G \times G$. Therefore, the maximum diversity gain is

$$
G_{d}^{\max }=M_{r} \max \left(\min _{\forall \mathbf{V}_{p}^{k} \neq \hat{\mathbf{V}}_{p}^{k}} \operatorname{rank}\left(\boldsymbol{\Theta}_{p}\right)\right)=G K M_{t} M_{r} .
$$

Note that $\mathbf{R}$ is of full rank if $G$ is less than the total number of multipath components $(C+1)(L+1)$. Due to the large bandwidth of UWB waveform, the received signal typically contains a significant number of resolvable multipath components. Consequently, the correlation matrix $\mathbf{R}$ is generally of full rank. Therefore, the maximum diversity order of $G K M_{t} M_{r}$ can be achieved by using a set of proper designed codeword matrices $\mathbf{V}_{p}$.

The result in (38) leads to some interesting observations as follows. First, the differential multiband UWB system achieves the same diversity gain under different channel environment. This implies that the clustering property of UWB channel does not strongly affect the diversity gain of differential multiband system. On the other hand, the coding gain which is a function of $\prod_{m=1}^{r} \lambda_{p, m}$ is severely affected by the multipath arrival rates and decay factors through the correlation matrix $\mathbf{R}$. Second, by incorporating the frequency-domain differential scheme with the multiband transmission, we are able to achieve the diversity gain of $G K M_{t} M_{r}$, regardless of the channel time-correlation property. This is different from the use of differential STF coding in the conventional MIMOOFDM systems, e.g. in [5], where the maximum achievable diversity gain is only $G M_{t} M_{r}$ due to the requirement of almost constant channels over several OFDM blocks.

\section{Simulation Results}

We performed simulations for a multiband UWB system with $N=128$ subcarriers and each subband occupies bandwidth of $528 \mathrm{MHz}$. The channel model parameters followed those for CM 1 and CM 2 [8]. The data matrix $\mathbf{V}_{p}$ in (9) were constructed by jointly coding across $G, K$, and $M_{t}$ using existing cyclic group codes [4]. In case of repetition based coding, the codeword is given by $\mathbf{V}_{p}=\mathbf{I}_{K} \otimes \mathbf{v}_{p}$, where $\mathbf{v}_{p}$ is a $G M_{t} \times G M_{t}$ jointly encoded diagonal matrix.

Figure 2 depicts the performances of single-antenna multiband UWB system with different number of $G$ and $K$. For fair comparison, the spectral efficiency is fixed at $R=1 \mathrm{~b} / \mathrm{s} / \mathrm{Hz}$ for all cases. The performances are simulated under CM 1. For uncoded differential system $(G=1$ and $K=1)$, we can see that the performance loss is more than $3 d B$ compared to the coherent detection, and an error floor can be observed. This is due to the effect of the channel mismatch between adjacent subcarriers. By jointly encoding across two OFDM symbols ( $G=1$ and $K=2$ ), the diversity gain is increased, hence resulting in significant performance improvement. As shown in Figure 2, the performance gain is more than $7 d B$ at the BER of $10^{-2}$. By further jointly encoding across two subcarriers $(G=2$ and $K=2)$, the proposed scheme obtains additional $4 d B$ gain at a BER of $10^{-3}$. This observation is in accordance with our theoretical result in (37) that the performance can be improved by increasing the number of jointly encoded subcarriers or the number of jointly encoding OFDM symbols. Moreover, at high SNR, the proposed jointly encoding differential scheme outperforms the uncoded multiband UWB system with coherent detection. We observe about $1-2 d B$ gain when $G=1$ and $K=2$, and about $3-5 d B$ gain when $G=2$ and $K=2$ at BER between $10^{-2}-10^{-3}$.

In Figure 3, we compare the performance of the proposed differential scheme under CM 1 and CM 2. The information is transmitted repeatedly across $K=1,2$, and 3 OFDM symbols, hence the transmission rate is $1 / K b / s / H z$. We can see that the performance of the proposed scheme under CM 1 is better than that under CM 2 for all cases. This is due to the fact that the multipath components in CM 2 are more random than that in $\mathrm{CM} 1$, which implies that compared with CM 1, CM 2 results in larger channel mismatch, and hence worse performance. For each channel model, the performance improves as the number of encoded OFDM symbols increases which confirms our theoretical analysis.

Figure 4 depicts the performances of differential UWBMIMO systems. The number of jointly encoded OFDM symbols is fixed at $K=1$, and the spectral efficiency is $R=$ $1 \mathrm{~b} / \mathrm{s} / \mathrm{Hz}$ for all cases. From Figure 4, we can observe the performance improvement as the number of antennas increases. When using two transmit and one receive antennas and encoding across one subcarrier and one OFDM symbol, the proposed scheme yields $7 d B$ improvement over the single antenna system. When we further jointly encode across two 


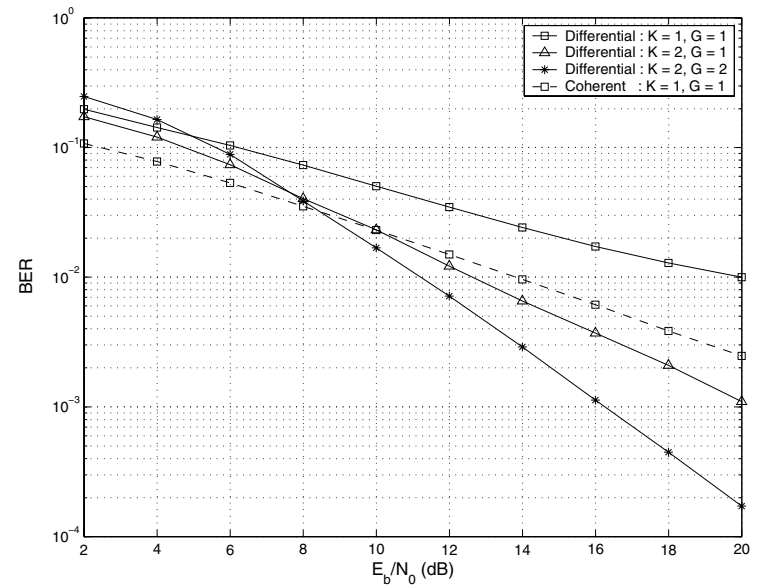

Fig. 2: Performance under CM1, $M_{t}=1, M_{r}=1, R=1 \mathrm{~b} / \mathrm{s} / \mathrm{Hz}$.

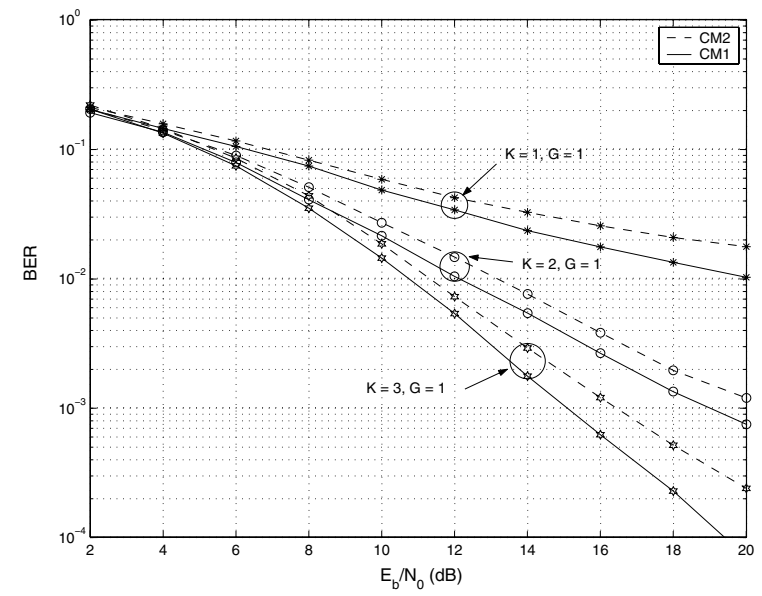

Fig. 3: Performance under $\mathrm{CM} 1$ and $\mathrm{CM} 2, M_{t}=1, M_{r}=1, R=$ $1 / \mathrm{K} b / \mathrm{s} / \mathrm{Hz}$.

subcarriers, additional performance gain of about $4 d B$ can be observed at a BER of $10^{-3}$. However, slightly error floors can still be observed when the data is encoded across multiple transmit antennas since the chance of channel mismatch is higher in this case. On the other hand, increasing the number of receive antennas improves the diversity gain without the tradeoff in the channel mismatch. In particular, an additional performance gain of $6 \mathrm{~dB}$ is observed when two receive antennas are employed.

\section{CONCLUSIONS}

We propose in this paper a frequency-domain differential scheme for multiband UWB systems. By a technique of band hopping in combination with jointly coding across spatial, temporal and frequency domains, The proposed scheme is able to explore the available spatial and multipath diversities, richly inherent in UWB environments. The analysis reveals that the proposed differential scheme achieves the same diversity advantage under different channel environments. However, the clustering behavior of UWB channels affects the performance through the coding gain. For single antenna multiband UWB system, simulation results show that the proposed differential multiband scheme yields superior performance to the conventional differential encoding scheme, particularly under very short-range line-of-sight scenario, e.g. in CM 1. We obtain

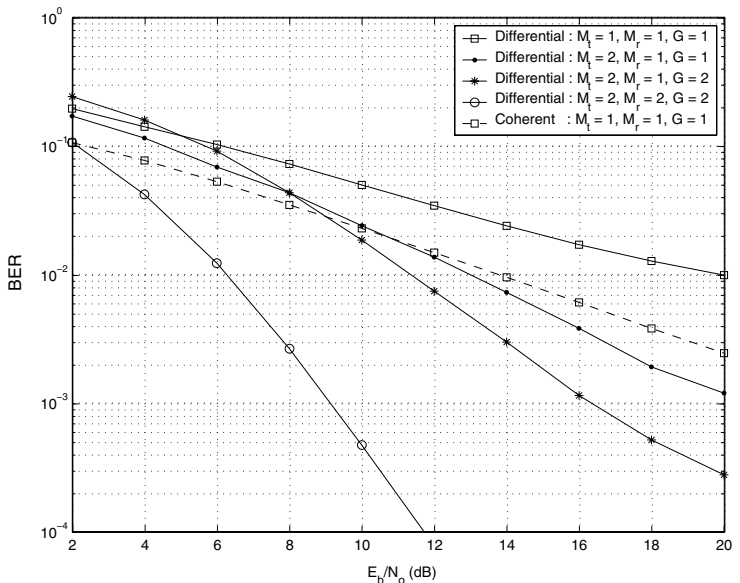

Fig. 4: Performance comparison of the proposed differential scheme under CM1 employing SISO and MIMO processing, $K=1$ and $R=1 \mathrm{~b} / \mathrm{s} / \mathrm{Hz}$.

about $7 d B$ gain at a BER of $10^{-2}$ when jointly encoding across one subcarrier and two OFDM symbols. Moreover, at high SNR range, the proposed jointly encoded differential scheme outperforms the uncoded coherent detection scheme of about $3-5 d B$ at BER between $10^{-2}-10^{-3}$. In case of multiband UWB system with multiple transmit antennas, while slightly error floor occurs due to the effect of channel mismatch, additional diversity can be observed when number of transmit antennas is increased. However, increasing the number of receive antennas improves the diversity gain without tradeoff in performance due to the effect of channel mismatch.

\section{REFERENCES}

[1] A. Batra, "Multi-band OFDM Physical Layer Proposal for IEEE P802.15 Task Group 3a," www.multibandofdm.org, Mar. 2004.

[2] M. Ho, V. S. Somayazulu, J. Foerster, and S. Roy, "A differential detector for an ultra-wideband communications system," IEEE Vehicular Technology Conf., vol. 4, no. 9, pp. 1896 - 1900, May. 2002.

[3] W. P. Siriwongpairat, W. Su, M. Olfat, and K. J. R. Liu "Spacetime-frequency coded multiband UWB communication systems," IEEE Wireless Commun. and Networking Conf., vol. 1, pp. 426-431, Mar. 2005.

[4] B. M. Hochwald and W. Sweldens, "Differential unitary space-time modulation," IEEE Trans. Commun., vol. 48, pp. 2041-2052, Dec. 2000

[5] Q. Ma, C. Tepedelenlioğlu, and Z. Liu, "Full diversity block diagonal codes for differential space-time-frequency coded OFDM," IEEE Global Telecommun. Conf., vol. 2, pp. 868-872, Dec. 1-5, 2003.

[6] T. Himsoon, W. Su, and K. J. R. Liu, "Single-block differential transmit scheme for frequency selective MIMO-OFDM systems," IEEE WCNC, vol. 1, pp. 532-537, Mar. 2005.

[7] W. Su and K. J. R. Liu,, "Differential space-frequency modulation for MIMO-OFDM systems via a smooth logical channel," IEEE Global Telecommun. Conf., Dec. 2004.

[8] J. Foerster, et. al, "Channel modeling sub-committee report final," IEEE802.15-02/490, Nov. 18, 2003.

[9] A. A. M. Saleh and R. A. Valenzuela, "A statistical model for indoor multipath propagation," IEEE J. on Selected Areas in Commun., vol. 5, no. 2, pp. 128-137, Feb. 1987.

[10] R. A. Horn and C. R. Johnson, Topics in Matrix Analysis, New York: Cambridge Univ. Press, 1994.

[11] K. L. Clarkson, W. Sweldens, and A. Zheng, "Fast multiple antenna differential decoding," IEEE Trans. Commun., vol. 49, pp. 253-261, Feb. 2001.

[12] M. Brehler and M. K. Varanasi, "Asymptotic error probability analysis of quadratic receivers in Rayleigh-fading channels with applications to a unified analysis of coherent and noncoherent space-time receivers," IEEE Trans. Inform. Theory, vol. 47, no.6, pp. 2383-2399, Sep. 2001.

[13] W. Pam Siriwongpairat, W. Su, K. J. R. Liu, "Characterizing performance of multiband UWB systems using Poisson cluster arriving fading paths," IEEE SPAWC, pp. 264-268, Jul. 2005. 